\title{
Germanica
}

\section{Jean Jourdheuil La Bataille D’Arminius de Kleist ou le récit de la forêt (Entretien avec Christian Klein)}

Propos recueillis par Christian Klein et en collaboration avec Michèle Jung (Montpellier)

\section{(2) OpenEdition}

\section{Journals}

Édition électronique

URL : http://journals.openedition.org/germanica/2053

DOI : $10.4000 /$ germanica. 2053

ISSN : 2107-0784

Éditeur

Université de Lille

Édition imprimée

Date de publication : 30 juin 1996

Pagination : 179-192

ISBN : 9782098426320

ISSN : 0984-2632

\section{Référence électronique}

Propos recueillis par Christian Klein et en collaboration avec Michèle Jung (Montpellier), « Jean Jourdheuil La Bataille D'Arminius de Kleist ou le récit de la forêt

(Entretien avec Christian Klein) », Germanica [En ligne], 18| 1996, mis en ligne le 14 juin 2013, consulté

le 06 octobre 2020. URL : http://journals.openedition.org/germanica/2053 ; DOI : https://doi.org/

10.4000/germanica.2053

Ce document a été généré automatiquement le 6 octobre 2020.

(c) Tous droits réservés 


\section{Jean Jourdheuil La Bataille D'Arminius de Kleist ou le récit de la forêt ${ }^{1}$ (Entretien avec Christian Klein)}

Propos recueillis par Christian Klein et en collaboration avec Michèle Jung (Montpellier)

\section{Christian KLEIN : Pourquoi cet intérêt pour Kleist?}

Jean JouRdHEuIL : J'ai vu le Prince de Hombourg par Peter Stein, quelques années plus tard, j'ai fait un $3^{\mathrm{e}}$ cycle sur Kleist où je me suis intéressé à deux choses. L'une qui était le cheminement de Kleist vers l'écriture, c'est-à-dire le fait que Kleist utilise la correspondance comme activité littéraire ou prélittéraire jusqu'au moment où il commence à écrire et où la correspondance devient fonctionnelle, c'est-à-dire que la dimension littéraire du travail s'est déplacé vers la littérature, le théâtre, etc. Vers 1802-1803 la correspondance n'est plus cette espèce de vocation littéraire. Ce travail m'a conduit à attribuer beaucoup d'importance à la crise kantienne, à savoir que si la vérité n'existe pas, il reste la volonté de l'individu isolé. De ce point de vue, Hermann est un personnage solitaire.

La $2^{\text {e }}$ chose que j'ai faite, c'est de commenter dramaturgiquement, de manière détaillée, deux textes : Penthésilée et Robert Guiscard le Normand.

Ensuite j'ai traduit quelques textes de Kleist avec J.-L. Besson. Je souhaitais monter La Bataille d'Arminius, en partie parce qu'elle a mauvaise réputation à cause des liens qu'elle entretient, de par sa réception, avec le nationalisme allemand. Aussi parce qu'elle fait coexister un imaginaire français et un imaginaire allemand de manière explicite, parce qu'elle a une dimension historique, là où d'autre pièces l'ont beaucoup moins.

Dans La Bataille, ce qui me paraît essentiel, c'est que sont abordés dans un contexte historique ancien des questions qui ensuite dans la littérature allemande ne seront abordés que sur le mode ludique chez Wagner, le côté ajointance et coexistence de la mythologie et de l'histoire dans La Bataille d'Arminius est une chose tout à fait singulière.

C'est une pièce historique par l'usage que Kleist fait des sources. Manifestement il a 
beaucoup lu, mais il fait aussi intervenir l'histoire de 1806-1809, l'histoire après Iéna. Il y a la dimension très importante de la religion des anciens Germains. Ce qu'André Robert avait traduit par village relève plutôt de la notion de campement. Je dirais que la mythologie germanique, c'est Wotan, les chênes et la forêt. La forêt est le pôle mythologique de cette chose, que Kleist traite de manière historique, c'est le lieu de la peur, la forêt des contes. La pièce est travaillée discrètement par la forme des contes. Les Germains sont des nomades et donc il ne fallait pas les sédentariser. Ce qui est très inquiétant pour les Romains. Quand les Romains arrivaient dans une forêt, ils coupaient des arbres pour faire des routes. Alors que les Germains se déplaçaient, ils se nourrissaient de cochons qui se nourrissaient de glands. Le nomadisme apparente les Germains aux Amazones. On retrouve Penthésilée, avec l'épisode de l'ourse. Les villages gaulois étaient sédentaires avec des structures mentales différentes.

Ce qui m'avait frappé à l'époque, c'était le problème de la guerre populaire pour les gens de ma génération : la guerre du Vietnam, Che Guevara. Peymann est de la même génération. Quand il a monté la pièce, il a fait de Hermann une figure de résistant, un Che Guevara, mais affublé d'une deutsche Hausfrau, Thusnelda ${ }^{2}$. La chose surprenante, pour moi qui a un point de vue différent, c'est de m'apercevoir qu'une chose qui semblait être l'apanage d'un mouvement de libération nationale pouvait être une sorte de pensée militaire définie en Allemagne pour mettre en échec Napoléon. Kleist n'a pas été le seul. Très clairement : c'est la guerre illégale, la guerre de partisans, ils avaient l'expérience de la guerre des partisans qui commençait en Espagne. Cette dimension anticoloniale est présente dans l'œuvre de Kleist, étrangement : Les Fiancés de Saint Domingue, par ex. On dit que ce n'est pas pour rien qu'il a vécu au fort de Joux, à quelques pas de la cellule où était Toussaint Louverture (mort en 1803). Kleist y était en 1807. Gauvain, l'ami de Kleist, aurait occupé la cellule de Toussaint Louverture. La question anticoloniale rejoint la question antinapoléonienne, dans la mesure où Napoléon a réintroduit l'esclavage aux Antilles.

Christian KLEIN : Curieusement le discours politique ne domine pas dans cette pièce que tu dis ne pas être un drame historique, ni une œeuvre de propagande (Tendenzdrama). Ce discours là est détourné. Comment vois-tu ce détournement, comment as-tu travaillé sur cette rupture?

Jean JouRDHEUIL: Il y a deux approches qui ont été importantes. L'une concerne le personnage de Hermann, c'est-à-dire le fait que ce personnage est seul. Kleist n'allait pas se refaire, sa façon de penser des héros décevants qui ne parvenaient pas à être des héros au moment où ils s'élèvent et pourraient nous élever avec eux, s'effondrent, s'isolent, s'enferment dans un moment de somnambulisme, qui fait que la pièce ne peut pas être historique, mais est nécessairement, de part en part, kleistienne. Ses héros sont eux-mêmes une énigme pour leur entourage. L'approche des pièces est donc l'approche de l'énigme. À chaque niveau d'approche on croit saisir l'énigme et on formule à son propos quelque chose qui est faux, donc on commet une méprise ou un malentendu, ce qui donne la possibilité à la pièce de progresser encore d'un cran pour passer du malentendu $n^{\circ} l$ au malentendu $n^{\circ} 2$ etc. et ainsi d'approcher l'énigme.

$\mathrm{Au}$ début, ce qui est surprenant dans la conversation de Hermann avec les Princes dans l'acte I, juste après la scène de la chasse, c'est qu'il dit : «je veux perdre, pour gagner je veux perdre comme il convient à un prince allemand ». 
Christian KLEIN : Cette incongruité a un effet comique sur le spectateur, en raison de la rupture dans le discours.

Jean JouRdHEUIL: Tous ces gens viennent le voir pour lui parler d'une chose simple : faisons alliance pour aller nous battre contre les Romains. Lui dit non, je ne fais pas alliance, parce que je veux perdre. En fait il ne veut rien partager. C'est là qu'on retrouve la crise kantienne. Et il n'y a pas de communication possible entre Hermann et les princes. Il y a entre eux un désaccord fondamental. Il veut se battre pour la liberté, les autres veulent se battre pour leurs troupeaux, leurs champs. Il est orienté vers l'être, les autres vers l'avoir. Et entre ces deux discours, celui de l'être et de l'avoir, il y a incompréhension totale.

Christian KLEIN : Et en même temps il y a un travail de satire politique. Ce personnage là est un non-lieu, il n'est pas là où il devrait être, pas là où on l'attend. II est un révélateur de la cacophonie qu'il y a autour de lui et des contradictions des princes allemands historiques, mais aussi des contemporains de Kleist. Dans le même ordre d'idées, il y a un autre passage où les généraux romains sont perdus dans la forêt. Leurs réflexions commentent et leur situation concrète et la situation générale.

Jean JouRDHEUIL : L'un dit : on n'y voit rien, le $2^{\mathrm{e}}$ dit : toute la terre qui nous colle aux pieds et le $3^{\mathrm{e}}$ dit : et en plus, cette langue on n'y comprend rien. Sur 3 registres :

- la visibilité par les yeux

- la marche par les pieds

- et l'écoute par les oreilles.

Ces trois textes successifs créent le degré d'égarement, le piège dans lequel sont tombés ces romains sur ces 3 plans.

Christian KLEIN : Ceci est saisi par les spectateurs comme comique et traduit par une mise en abyme le désarroi qui domine cette pièce, la difficulté à s'orienter, la difficulté à avoir des repères précis, y compris dans les mots et la façon dont un discours qui doit orienter quelqu'un est d'entrée de jeu l'objet de malentendus et ramène le personnage, Varus, à son point de départ. À l'intérieur de cette pièce il y a toute une série de micro-dialogues qui sont autant de mises en abyme de se qui se passe au niveau de l'ensemble de la pièce, par exemple l'ours de Thusnelda: la scène prend au pied de la lettre l'expression "il a fait de moi une ourse », et la bête devient réellement une bête, et dévore l'ennemi sans merci.

Jean JouRDHEUIL: Tout à fait. Le texte est comme la forêt. C'est elle qui raconte l'histoire, elle est le lieu d'énonciation. L'énonciation est aussi heurtée et bégayante que la vision est gênée dans une forêt.

Christian KLEIN : Au sujet de la diction des acteurs. II y a dans ton spectacle un parti pris déclamatoire pour la plupart des personnages, sauf Arminius - en contraste avec son attitude assez sereine, calme, il fait preuve d'une certaine assurance, voire d'une certaine aménité. Ventidius, par exemple, pourrait jouer l'amant déçu, le héros racinien, ou un personnage double ce qu'il est - mais une personne clivée qui ne maitriserait pas son clivage. II est à la fois un intrigant et un amoureux, et il a une mission politique.

Jean JoURDHEUIL : Il y a effectivement une façon de les traiter dans ce contexte là comme des personnes «classiques" à la Schiller. C'est-à-dire qu'ils sont, eux, naturellement des « héros ». Et du coup, quand ils ont une arrière-pensée, ça se voit tout de suite. Elle est flagrante. Ils dissimulent le fait que les Romains jouent un jeu diplomatique qui n'est pas d'une franchise absolue. Ils ont des arrière-pensées. Une fois que Marbod aura été vaincu, ce sera le tour de Hermann, et après avoir vaincu l'un et l'autre, le pouvoir des Romains sera instauré en Germanie. Les Romains sont l'hypocrisie élémentaire des métropolitains dans un contexte colonial. Cette hypocrisie a des aspects qui pourraient être à la Titus et Bérénice et dans le même 
temps des aspects qui confinent au vaudeville, lorsque Varus par exemple offre la petite panoplie de Cupidon, les parfums, les plumes etc. Il est clair qu'on se livre là à une moquerie à l'égard des Français et de la mode. C'est aussi la façon qu'ont les coloniaux de traiter les indigènes et de leur mentir, de les prendre pour des imbéciles, pour des enfants, des sous-hommes. C'est vrai que la vision de Kleist mêle les deux registres. Cela concerne Ventidius au début et Varus à la fin. Il y a une troisième personne, Septimius, qui dit clairement, moi qui ai vaincu le lignée des hommes royaux en Germanie je finis déchiré par des chiens, par des gens qui « sont indignes d'être des héros » (acte $V$, scène 13). C'est-à-dire qu'ils se définissent euxmêmes comme des héros. La problématique kleistienne du héros qui est un antihéros rejoint des remarques de Tacite sur le caractère problématique d'Arminius comme héros. Il dit, ça aurait pu être un héros, mais curieusement ça n'en est pas un.

Christian KLEIN : Pourquoi monter Kleist après avoir monté Müller?

Jean JouRDHEUIL : La pièce elle-même intéresse Müller. Chez l'un comme chez l'autre le récit est autre chose que la fable. La dimension de récit acquiert une très grande autonomie dans le théâtre de Müller. Certains textes de théâtre de Müller se présentent comme des récits, ils citent des scènes, les font surgir et les congédient. $L a$ Bataille (Die Schlacht) commence par un récit, qui fait surgir la scène des deux frères, puis la scène est suspendue de nouveau par un moment de récit, puis de nouveau... etc. Dans La Bataille il y a plusieurs scènes comme ça, mais aussi dans La Route des Chars : ça se présente comme des récits, c'est-à-dire ce que le théâtre selon Aristote faisait disparaître, le récit, pour ne laisser que les scènes dialoguées et la mimesis. Chez Müller, il y a au contraire une autonomie du récit, ce qui lui permet de quitter le registre de l'action comme registre principal pour rendre plus importantes les questions de l'espace-temps. Le même problème existe chez Kleist : chez Kleist les personnages font très souvent des récits, des scènes d'interrogatoire, de mises à l'épreuve. Cela donne un régime du texte qui est très différent. Toute scène dialoguée intervient toujours par rapport à la précédente. Elle prend l'action là où elle est arrivée pour la continuer. Les interrogatoires, par contre, sont toujours repris depuis le début, c'est-à-dire que le deuxième interrogateur ne prend pas la suite du précédent, ce qui donne un régime très particulier au texte de théâtre chez Kleist. Cela renvoie à ce que tu disais tout à l'heure sur la mise en abyme. Les séquences micro-cosmiques : sur une séquence, c'est comme ce qui se passe sur l'ensemble de la pièce. Ça renverrait, en mathématiques modernes au fractal, par prolifération, ce qui est petit devient ce qui existe en grand.

Christian KLEIn : Il y a un rapport à la logique du rêve. Par exemple chez Kafka, le Verdict est construit sur la logique du rêve. Quel que soit le scénario, on arrive au même résultat. Parce qu'au départ, il y a un problème qui n'a pas de solution dans la réalité, autre qu'imaginaire en permutant les rôles. On peut varier les scénarios, on retrouve toujours la même non-solution entre le mariage et/ou l'écriture. Pour quelqu'un comme Kleist, depuis la crise kantienne de 1800-1801, il n'y a plus de projet d'existence: on ne peut pas construire son existence sur la recherche du bonheur absolu. Qu'est-ce qui reste: toute une série de scénarios qui sont interchangeables. II n'y a pas quelque part une vérité qu'il s'agirait de découvrir, mais des situations particulières.

Jean JOURDHEUIL : Oui, ces trois auteurs ont cela en commun. Müller s'intéresse à Kafka qui s'intéresse à Kleist. Kleist multiplie le récit à l'intérieur d'un schéma mimétique, comme s'il minait ou vidait la mimesis de l'intérieur. L'action, qui n'est saisissable qu'au niveau du langage, devient action intérieure, une approche de l'énigme qui est 
déposée dans le caractère de Hermann. C'est de l'intérieur du schéma mimétique qu'il fait proliférer les récits. Alors que Müller, lui, présente d'emblée le registre du récit qui suscite et congédie la représentation mimétique, mais l'un comme l'autre aboutissent à faire tomber l'action qui était la pièce centrale de la politique du théâtre depuis Aristote, brusquement elle n'est plus la chose principale sur quoi tout doit se régler. C'est pourquoi l'espace et le temps deviennent importants. Par exemple, dans le Ve acte, il y a une chose qui est extrêmement difficile : la scène des Romains dans la forêt qui découvrent qu'ils sont encerclés par Marbod et par Arminius, la scène de Hermann qui envoie des signaux, qui reçoit des signaux de Marbod et qui doit régler le problème d'une rébellion de ses soldats et qui doit donner le plan de guerre en disant à ses soldats, c'est pas contre Marbod que l'on se bat, mais contre les Romains, la scène de Thusnelda avec l'ourse qui mange Vintidius. Ces trois scènes ont lieu en même temps, or elles sont présentées successivement dans la pièce. Et elles ont lieu toutes les trois au petit matin, au moment où l'aube point. Ça signifie qu'on n'est pas dans la représentation d'une action, parce que ce sont au fond trois actions simultanées en trois endroits différents. On s'en tire avec une petite astuce : le rouge de l'aurore, qui ensuite augmente, puis on redémarre avec la même lueur rouge. Cela signifiait que la question de l'espace-temps devient l'objet de la représentation, et non plus l'action elle-même. Le spectateur ne peut pas utiliser son réflexe habituel de spectateur, à savoir que l'action progresse quand une scène succède à une autre. Là ce n'est pas le cas.

On tombe là sur des problèmes de la théorie de la connaissance, le problème d'Amphitryon : comment être à deux endroits au même moment.

Christian KLEIN: Tu as utilisé la forme du procès pour monter le spectacle, comme une radiographie de l'événement...

Jean JOURDHEUIL: La radiographie fait voir ce qui ne se voit pas. On retrouve la forme de l'interrogatoire : j'ai quelque chose à te dire - qu'est-ce que tu as à me dire ? Ça n'est jamais un dialogue avec un échange à égalité.

Christian KLEIN : L'autre répond par des faux-fuyants, l'interrogateur a du mal à obtenir des renseignements...

Jean JOURDHEUIL : Oui, soit parce que celui à qui la question est posée ne sait pas, il a $\mathrm{vu}$, mais il n'a pas pensé que c'était cela qu'il devait regarder. Il a entendu, mais il n'a pas pensé à noter dans sa mémoire, donc il faut que lui-même la reconstitue. On n'a pas affaire à des personnages dotés d'une psychologie comme chez Ibsen. Ce qui constitue les personnages, c'est le récit qu'ils se tiennent par cette structure d'interrogatoire ou de procès. En fait, il y a une formidable ténuité des figures, parce qu'ils ne sont pas des choses positives, mais des énigmes les uns pour les autres. Ils sont des effets de récits, des effets d'interrogatoire. C'est l'interrogatoire qu'Hermann fait subir à Thusnelda qui fait jaillir qui est Thusnelda, qui l'oblige à se dévoiler. Il y a des structures de dévoilement progressif, et ce qui se dévoile de manière progressive, c'est ce qui se passe au niveau intérieur, c'est le spectateur qui crée cette action à partir de tous les éléments qui lui sont proposés. Goethe avait bien raison quand il parlait de théâtre invisible. 
Christian KLEIN : /l y a des choses qui échappent au spectateur. Par exemple. On ne saura jamais si la lettre est une fabrication d'Hermann ou si Vintidius est aussi fourbe qu'il apparaît. II y a une relative indétermination.

Jean JOURDHEUIL : Il est possible que la lettre soit de Vintidius, comme il est tout à fait possible qu'Hermann l'ait inventée pour arriver à ses fins. Les deux choses sont possibles. Quand Hermann dit: il me faut des Germains déguisés en Romains pour qu'ils commettent des meurtres, et puis ensuite il y a le viol de la jeune Hally. Qui estce qui a commis le crime? Est-ce que ce sont des Germains déguisés en Romains? Faut-il croire Hermann quand il arrive tout étonné et dit : je sors de ma tente, je ne sais rien?

Le théâtre invisible est un théâtre maximum d'imagination par reconstitution, procès, récit etc. Cette notion d'invisibilité, Goethe l'avait bien vu, n'allait pas avec la représentation de l'action. La représentation positive de l'action est problématique et donc ne se laisse pas voir. Ici la forêt joue le jeu des Germains. Les Germains ne se voient pas dans la forêt. En revanche les Romains en uniforme bleu et blanc se voient effectivement tout de suite dans la forêt. Ce qui n'est pas vu, l'invisible, est la condition du visible. Pour voir, il faut que quelque chose ne soit pas vu.

Il y a aussi un anachronisme chez Kleist que nous avons prolongé. Varus devient un peu napoléonien et aussi mussolinien, Vintidius devient lui aussi un peu D’Annunzio, c'est une façon d'aller jusqu'à la première guerre mondiale. C'est comme ça que les Romains se retrouvent avec les side-cars, c'est la motorisation de l'armée allemande. Dans les uniformes des Romains, il y a quelque chose de la Wehrmacht. Les Romains sont un formidable patchwork qui va de Napoléon à Mussolini et la Wehrmacht, alors que les Germains ont des côtés princes arabes ou asiatiques. Il y a des bruits de chars très récents. Cette logique de l'anachronisme contribue aussi à sortir la pièce de sa dimension strictement historique.

Christian KLEIN : Ça superpose des couches historiques.

Jean JouRDHEUIL : Voilà, c'est la même chose que les mises en abyme des petites scènes qui reprennent la même problématique que l'ensemble. Chez Kleist il n'est pas naturel de parler par métaphore, à la différence de Shakespeare. C'est pourquoi le récit est plus important que cette représentation enrobée de métaphores.

Christian KLEIN : Tu as trouvé un ton qui maintient le discours de Kleist à un certain niveau du sol pour que le spectateur ait toujours cette distance, les différents éléments avec laquelle ii fabrique son discours. À aucun moment on ne plonge dans une action ou on n'oublie l'origine de ce discours, on n'oublie qu'il est construit.

Jean JouRDHEUIL : Il fallait retrouver cette intuition des possibilités de raconter et de représenter, sinon on fait un téléfilm.

Christian KLEIN : Hermann est-il un héros pervers, sur le modèle de Michael Kohlhaas?

Jean JouRDHEUIL: Je crois qu'il est comme Michael Kohlhaas. Au début il est dans la situation de quelqu'un qui a subi une injustice, c'est-à-dire la conquête de son pays. Il subit une injustice et construit sa révolte, son opposition, qui est tout à fait légitime. Et ensuite, de fil en aiguille, ça devient d'une certaine manière une chose tout à fait monstrueuse.

Christian KLEIN : Et il se passe quelque chose de tout à fait étrange, au moment où il devient un héros, tu le montres très bien, il est allongé.

Jean JouRDHEUIL : J'ai d'abord essayé de voir les désaccords qu'il y a entre eux et le fait que Hermann n'a pas la majorité. C'est Marbod qui a la majorité et qui contrôle la 
structure du pouvoir politique. Et Hermann, de nouveau, est isolé, il ne sait pas quoi faire, il est hésitant. Il a fini. Il doit faire quelque chose et on lui confie le sort d'Aristan, le chef des Ubiens, c'est-à-dire de Cologne et des princes rhénans, qui tient un discours sur la Germanie: que sais-je de la Germanie, je suis prince d'un Etat souverain et je peux m'allier avec qui je veux, y compris avec Varus. Le discours sur la Germanie fait manifestement allusion à deux poèmes de Goethe et de Schiller dans lesquels l'Allemand ne doit pas être national mais un homme international. La réponse de Hermann est à certains égards le réponse de Kleist à Goethe et à Schiller : il fait décapiter Aristan, comme ça il saura où est la Germanie. C'est peut être, à certains égards, Kleist décapitant Goethe et Schiller. Brusquement sa volonté, qui était individuelle, devient une volonté politique et collective. Kleist est le prototype du Junker délinquant et poète, sans doute l'un à cause de l'autre. Et cette délinquance fait qu'au moment où on donne le pouvoir à Hermann, ça devient catastrophique, et là il $\mathrm{y}$ a une intervention de la mise en scène, c'est le moment où il se couche et où il dit ces choses horribles sur la destruction de Rome. Mais il le dit comme le rêve de quelqu'un qui avait une position identique juste avant la bataille. Il est arrivé à ses fins, il a bouté dehors les Romains. Plus rien n'existait que sa volonté. Au moment où il a gagné, il a tout sacrifié et donc tout perdu : il se fait battre bêtement en duel. Ce n'est pas lui qui remporte la victoire, ni qui tue Varus. Tout lui échappe.

Christian KLEIN : C'est la ruse de Kleist. II fait un pied de nez ironique.

Jean JouRDHEUIL: Il sabote le happy end. Chaque fois qu'on représentait cette pièce en Allemagne avec des fins nationalistes, on coupait des scènes jugées « inadmissibles ». Peymann a fait des coupes à son tour, mais pour sortir la pièce de son purgatoire nationaliste. Grâce à lui, la pièce est redevenue fréquentable et ma mise en scène est un dialogue avec celle de Peymann. Je m'efforce de la contredire sur certains points. C'est cela qui fait qu'une culture européenne puisse exister. Par exemple, en faisant des Germains des nomades, et pas du tout des personnages wagnériens. Peymann a fait une parabole sur la guerre populaire. Il n'a pas laissé le jeu des interrogatoires, ce qui est en coquetterie avec la déception des spectateurs. Il a brechtisé et shakespearisé, mais il a coupé environ un quart du texte. Nous avons assez peu coupé. Il y a quelques condensations.

Christian KLeIN : Et Gilles Aillaud?

Jean JoURDHEUIL: Je travaille avec Gilles Aillaud depuis 1972, en rupture avec la voie magistralement ouverte par René Allio. Il s'agissait ici de faire du décor un lieu d'énonciation, donc un espace acoustique. Le décor prend une dimension métaphorique, comme lieu espace-temps. Les acteurs ne jouent pas devant, mais dans la forêt. Sinon, on tombe dans le registre de la parabole. Or Kleist ne fonctionne pas dans ce registre, mais dans celui du dévoilement progressif d'une action à caractère énigmatique. D'où le choix de ce lieu unique où les arbres bougent pendant la représentation. Ainsi, par exemple, la première apparition de Varus. Il n'avance pas, un arbre se déplace et le fait apparaître, c'est-à-dire que - au pied de la lettre - il est déjà là. Le décor n'illustre pas. Platon dit que le poète n'a pas le droit de se cacher, il doit être responsable de son énonciation. C'est la forêt qui peut raconter, qui survit à l'histoire. Il s'agit de revenir sur les lieux, comme le faisaient les Romains de Germanicus, de représenter par le truchement de gens qui sont morts (et non d'une mimesis par imitation). 
Christian KLeIn: Hermann est ce que n'avait pas pu être Kleist: celui qui maîtrise toutes ses émotions, sauf une fois. Kleist s'approprie un mythe national par l'écriture. II essaie de régler un problème personnel en en montrant le fragilité.

Jean JouRDHEUIL: À travers ces récits les personnages acquièrent une liberté potentielle par rapport à leur auteur. L'auteur n'instrumentalise pas Hermann comme étant le narrateur fictif. Dès lors qu'il a mis la machine narrative en route, elle l'entraîne plus loin : il y a un goût de Kleist pour les maths. Les pièces sont construites algébriquement. Il y a une construction mathématique pour tenir l'émotion, mais qui n'essaie pas de dissimuler qu'il s'agisse d'une construction, et qui laisse de temps en temps l'action s'effondrer. Ça passe par des syncopes.

\section{NOTES}

1. Cet entretien a été effectué en mars 1995 à Paris à l'occasion de la mise en scène par Jean Jourdheuil de la pièce de Kleist La Bataille d'Arminius, traduction Jean-Louis Besson et Jean Jourdhueil, décors et costumes : Gilles Aillaud, Stefanie Bürkle. Le texte est publié aux Éditions Théâtrales, Paris 1995.

2. Peymann a monté la pièce en mars 1985 à Bochum. 